\title{
Hysteretic dynamics of domain walls at finite temperatures
}

\author{
T. Nattermann ${ }^{1}$, V. Pokrovsky ${ }^{2,3}$ and V.M. Vinokur ${ }^{4}$ \\ ${ }^{1}$ Institut für Theoretische Physik, Universität zu Köln, D-50937 Köln, Germany \\ ${ }^{2}$ Department of Physics, Texas A $6 M$ University, College Station, TX 77843-4242, USA \\ ${ }^{3}$ Landau Institute for Theoretical Physics, ChernogolovkaMoscow District, 142432, Russia \\ ${ }^{4}$ Argonne National Laboratory, Argonne, IL 60439, USA
}

(November 3, 2018)

\begin{abstract}
Theory of domain wall motion in a random medium is extended to the case when the driving field is below the zero-temperature depinning threshold and the creep of the domain wall is induced by thermal fluctuations. Subject to an ac drive, the domain wall starts to move when the driving force exceeds an effective threshold which is temperature and frequency-dependent. Similarly to the case of zero-temperature, the hysteresis loop displays three dynamical phase transitions at increasing ac field amplitude $h_{0}$. The phase diagram in the 3 -d space of temperature, driving force amplitude and frequency is investigated.
\end{abstract}

Pinning dominated driven dynamics of elastic media in random environment is a paradigm for a vast diversity of physical systems. Examples include vortices in type II superconductors, charge density waves (CDW) in solids, stripe phases, Wigner crystals, dislocations in crystals, domain walls in magnets and many others [1]. Having appeared first in the context of dislocation dynamics [2], the scaling theory of glassy dynamic state of random elastic media came to fruition in the context of CDW [3] and vortex lattices in high temperature superconductors [4, 3 , and enjoyed an impressive success in explaining a wealth of phenomenology of the low temperature vortex state [5]. A closely related subject is the zero temperature depinning transition first studied for CDWs [6,7] and domain walls [8] 9 . Despite the significant recent progress, several key questions specific to glassy dynamics are yet poorly understood. One of such fundamental key issues, although known and extensively studied for more than hundred years in magnets, is hysteresis of interfaces subject to the applied ac drive and related aging and memory effects. A quest for urgent progress in understanding hysteretic behavior of magnetic domain walls is motivated also by emerging technological nano-scale magnetic systems whose ac properties are controlled by the hysteretic dynamics of interfaces.

A step towards theoretical description of hysteretic behavior of disordered interfaces has been undertaken in [10], where the cyclic motion of the domain wall at zero temperature under the ac field was investigated and the resulting magnetization hysteretic loop was described. A finite temperature may change drastically the interface dynamics: thermally activated creep motion becomes possible at any small drive.

In this Letter we develop a unified description of thermally activated and over-threshold domain wall dynamics in impure magnets. We demonstrate that at finite temperature new scales of length, activation energy and force appear leading to emergence of a new, temperature and frequency-dependent threshold field in the case of ac drive. The latter is the first in a series of dynamical phase transitions. To be specific, we will speak on magnetic domain walls. Accordingly we will be using either of terms "force" or "field" equivalently.

Finite temperature dc dynamics The essential of the zero-temperature dynamic behavior of an elastic medium in a random environment is the existence of the finite threshold depinning force $h_{p}$, separating immobile at $h<h_{p}$ and sliding at $h>h_{p}$ states of the system. Near the threshold the sliding velocity $v$ shows a critical behavior [6] $v \sim\left(h-h_{p}\right)^{\beta}$. At finite temperatures and $h \ll h_{p}$ thermally activated drift motion controlled by the static rugged energy landscape occurs. The latter is governed by the interface free energy

$$
\mathcal{H}=\int d^{D} x\left\{\frac{1}{2} \Gamma(\nabla Z)^{2}+V(\mathbf{x}, Z)-h Z(\mathbf{x})\right\}
$$

where $\Gamma$ is the interface stiffness, $h$ is the external driving force and $V(\mathbf{x}, Z)$ is the random impurity potential. $D$ dimensional vector $\mathbf{x}$ is the coordinate along the interface, and $Z$ is the coordinate of the transverse interface displacement. In the following we assume that the disorder average of the random potential vanishes. There are two different types of impurities, random bond (RB) and random field (RF) type in terms of magnetic models. The RB potential obeys the Gaussian statistics with:

$$
\overline{V_{R B}(\mathbf{x}, Z) V_{R B}(\mathbf{0}, 0)}=v^{2} l^{D+1} \delta(\mathbf{x}) \delta(Z)
$$

where $v^{2}=v_{0}^{2} c . v_{0}, c$ and $l$ denote the strength, the concentration and the correlation length of the impurity potential. In the RF case $V_{R F}(\mathbf{x}, Z)=\int_{0}^{Z} h\left(\mathbf{x}, Z^{\prime}\right) d Z^{\prime}$ where the $\mathrm{RF} h(\mathbf{x}, Z)$ has properties similar to $V_{R B}(\mathbf{x}, Z)$.

The static interface in a random environment becomes rough. Its roughness obeys the scaling law [13]: 


$$
w^{2}(L)=\overline{(Z(\mathbf{x})-Z(\mathbf{0}))^{2}} \approx l^{2}\left(\frac{L}{L_{p}}\right)^{2 \zeta} ; L=|\mathbf{x}|
$$

where the roughness exponent is $\zeta=\frac{4-D}{3}$ for $\mathrm{RF}$ and $\zeta \approx 0.2083(4-D)$ for $4-D \ll 1$ and $\zeta=2 / 3$ for $D=2$, for RB impurities, respectively 14.13]. The rough configuration develops over length scales $L \geq L_{p}$, where the Larkin length $L_{p}$ is a distance at which a typical fluctuation of the pinning forces, balanced by elastic forces, produces the transverse displacement $w \sim l$ :

$$
L_{p} \approx l(\Gamma / v)^{2 /(4-D)} .
$$

Note that the typical slopes $w(L) / L$ of the wall vanish for $L \gg L_{p}$ since $\zeta<1$. The energy barriers which must be overcome to depin a segment of the wall with the linear size $L$ is [2]:

$$
E_{B, 0}(L) \approx T_{p}\left(L / L_{p}\right)^{\chi} ; \chi=D-2+2 \zeta .
$$

Here $T_{p} \equiv E_{B, 0}\left(L_{p}\right) \approx \Gamma l^{2} L_{p}^{D-2}$ is a typical pinning energy on a scale $L_{p}$. At temperature $T>T_{p}$ the effective force necessary for depinning drops rapidly with the temperature 22. If an external driving force $h$ is applied, the total energy barrier $E_{B}(L)$ becomes

$$
\begin{aligned}
E_{B}(L) & \approx E_{B, 0}(L)-h L^{D} w(L) \\
& \approx E_{B, 0}(L)\left(1-\left(L / L_{h}\right)^{2-\zeta}\right)
\end{aligned}
$$

where $L_{h} \approx L_{p}\left(h_{p} / h\right)^{1 /(2-\zeta)}$. The energy barrier reaches its maximum $E_{B, \text { max }}$ at $L \approx\left(\frac{\chi}{D+\zeta}\right)^{1 /(2-\zeta)} L_{h}$ which gives $E_{B, \max } \approx T_{p}\left(h_{p} / h\right)^{\mu}$ and $\mu=\chi /(2-\zeta)$.

For completeness we note here that equilibrium RF systems in $d=D+1=2$ bulk dimensions have no long range order at length scales $L \gg \xi_{R F} \approx l \exp c(\Gamma / v)^{4 / 3}$ [16]. For weak disorder $\xi_{R F}$ is very large and can easily exceed the system size. As soon as an external field is applied such that $L_{h}<\xi_{R F}$, domain wall motion is dominated by the forces on scales $L_{h}$ and the absence of true long range order can be ignored.

As first found by Middleton 17], close to $h_{p}$ the smallest energy barrier vanishes as $\left(h-h_{p}\right)^{\theta}$ with $\theta=2$. We therefore get the effective energy barrier at $h \lesssim h_{p}$ if we replace in the above expressions $T_{p}$ by $\tilde{T}_{p}=T_{p}\left(\frac{h_{p}-h}{h_{p}}\right)^{\theta}$.

The time scale to overcome this barrier is of the order $\tau\left(L_{h}\right) \approx \tau_{0} \exp \left(\tilde{E}_{B, \max } / T\right)$, where $\tau_{0}$ is a microscopic hopping time, which leads to an average velocity:

$$
v(h) \approx \gamma h \exp \left[-\frac{T_{p}}{T}\left(\frac{h_{p}-h}{h_{p}}\right)^{\theta}\left(\frac{h_{p}}{h}\right)^{\mu}\right]
$$

where $\gamma$ is the effective friction coefficient [2, 3, 15].

At low temperature $T \ll T_{p}$ the dynamic threshold $h_{p}$ separates the creep regime from the active sliding regime. As can be seen from (7) a characteristic crossover field $h_{T}$ plays the role of the depinning force, where

$$
\frac{h_{T}}{h_{p}}=\left(\frac{\tilde{T}_{p}\left(h_{T}\right)}{T}\right)^{1 / \mu}=\left(\frac{T_{p}}{T}\left(1-\frac{h_{T}}{h_{p}}\right)^{\theta}\right)^{1 / \mu}
$$

At $h \approx h_{T}$, the drift velocity increases rapidly and at larger fields it displays almost linear behavior $v \approx \gamma h$. Note that $h_{T}$ is a monotonously decreasing function of temperature with a maximum $h_{T}=h_{p}$ at $T=0$.

In a close vicinity of the threshold field $h_{p}$, the effective energy barrier becomes small and even small thermal fluctuations may be sufficient to overcome it. At finite temperatures and $h \lesssim h_{p}$ the wall moves via thermal activation process with velocity given by eq. (7). Strictly speaking, it means that at finite temperatures the critical point shifts from $h=h_{p}$ to $h=0$. Yet there remains a memory of the critical behavior around $h \approx h_{p}$ displaying itself in a crossover behavior at finite but low temperatures. The crossover is seen as a rounding of the $h-v$ characteristics $v\left(h \approx h_{p}\right) \sim T^{\beta / \theta}$. We now can write an interpolation formula for the velocity which is valid in a wide range of variables:

$$
v(h, T)=\gamma h F(x, y) ; x=h / h_{p} ; y=T_{p} / T,
$$

$$
\begin{aligned}
F(x, y)= & \frac{\Theta(1-x)}{1+\left(y x^{-\mu}\right)^{\beta / \theta}} \exp \left[-y x^{-\mu}(1-x)^{\theta}\right] \\
& +\Theta(x-1)\left[\frac{1}{1+\left(y x^{-\mu}\right)^{\beta / \theta}}+\left(1-\frac{1}{x}\right)^{\beta}\right]
\end{aligned}
$$

Here $\Theta(x)$ is the step function equal to zero at $x<0$ and equal to 1 at positive $x$. The interpolation formula (10) satisfies following requirements: (i) $v(h, T)=$ $\gamma h$ at any fixed $T$ and $h \gg h_{T}$; (ii) $v(h, T)=$ $\gamma h \exp \left[-\frac{T_{p}}{T}\left(\frac{h_{p}-h}{h_{p}}\right)^{\theta}\right]$ for $h_{p}-h \ll h_{p}$ and $T \ll T_{p}$; (iii) $v(h, T) \approx \gamma h \exp \left[-\frac{T_{p}}{T}\left(\frac{h_{p}}{h}\right)^{\mu}\right]$ for $T \ll T_{p}, h \ll h_{p}$ and $E_{B, \max } / T \gg 1$; (iv) $v(h, T) \approx \gamma h_{p}\left(h / h_{p}-1\right)^{\beta}$ for $\left(h / h_{p}-1\right) \ll 1$ and $T \ll T_{p}\left(h / h_{p}-1\right)^{\theta} ;(\mathrm{v})$ $v\left(h_{p}, T\right) \approx \gamma h_{p}\left(T / T_{p}\right) \beta / \theta$ for $T \ll T_{p}$.

So far we assumed that the propagating interface is self-affine. This is confirmed by numerical simulations in $D>1$ interface dimensions for systems with weak disorder [18]. In $D=1$ dimensions the situation is less transparent: in simulations which use a bounded distribution of random fields the interface appears to be self-affine [19] or faceted 20] depending on whether lattice effects are avoided or admitted, respectively. We ignore here the possibility of faceted growth which occurs only in systems with narrow magnetic domain walls. For an unbounded distributions of random fields however a percolative selfsimilar domain wall propagation was observed [21]. In the following we will always assume, that the random fields distribution is bounded such that the domain walls 
remain well defined. This is also confirmed by our earlier simulation outside of the critical region 10.

Alternating fields. If the external drive is oscillating with frequency $\omega, h=h_{0} \sin \omega t$, the barriers for which $\omega \tau(L)>1$ cannot be overcome during one cycle of the ac field. From the condition $\omega \tau=1$ we find a new frequency and temperature dependent magnetic field $h_{\omega}$ which obeys

$$
\frac{h_{\omega}}{h_{p}}=\left(\frac{T_{p}}{T \Lambda}\left(1-\frac{h_{\omega}}{h_{p}}\right)^{\theta}\right)^{1 / \mu}
$$

where $\Lambda=\ln 1 /\left(\omega \tau_{0}\right)$. $h_{\omega}$ plays the role of the dynamic threshold. At low fields $h_{0}<h_{\omega}$ there is no macroscopic motion of the wall, its segments oscillate between the metastable states with close energies giving rise to dissipation [2]. Drift of the wall starts at $h_{0}>h_{\omega}$. We assume $\omega \tau_{0} \ll 1$, so that $h_{\omega}<h_{T}$. Various regimes of domain wall motion are summarized in Fig.1.

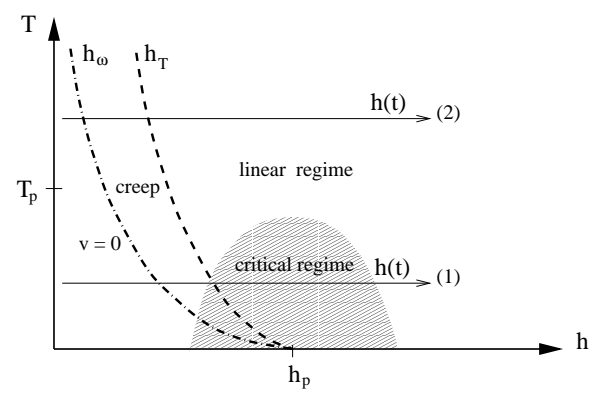

FIG. 1. The phase diagram for the domain wall hysteresis at finite temperature and frequency. Solid and vertical lines separate no sliding, thermal creep and mechanical drift regimes. See also explanations in the text.

Having derived the domain wall velocity as a function of the driving field in the different $h-T$-regions we consider now the magnetic hysteresis following from the motion of a single wall under the influence of an oscillating field. Since substantial length scales are larger than $L_{p}$, where slopes are small, the domain wall will be considered as a straight line (plane) characterized by one coordinate $Z$ [10]. Its dynamics is determined by equation of motion

$$
\dot{Z}=v(h(t))
$$

$Z$ varies between limiting values 0 and $L$. Here $L$ is the linear size of the sample in the case of a single domain wall or, in the multi-domain case, equal to the average distance between expanding nuclei . For harmonically oscillating field $h=h_{0} \sin \omega t$, equation (12) can be rewritten in terms of $h$ only:

$$
\frac{d Z}{d h}=\frac{v(h)}{\omega \sqrt{h_{0}^{2}-h^{2}}} ; \quad Z(h=0)=0
$$

Equations (12) and (13) are valid for $h>h_{\omega}$. The field region $h<h_{\omega}$ where the motion has zero drift velocity will not be considered here. The value $h_{\omega}$ plays the same role as the threshold field $h_{p}$ plays at zero temperature. At $h>h_{\omega}$ the hysteresis is dominated by the activation processes. In this respect it is similar to the nucleation dominated hysteresis described in [10] with two essential differences. First, the activation relates to the formation of a nucleus on the interface, not in the bulk. Second, the activation energy depends on the magnetic field as a power function $\sim h^{-\mu}$ due to the distribution of barriers depending on their length scale.
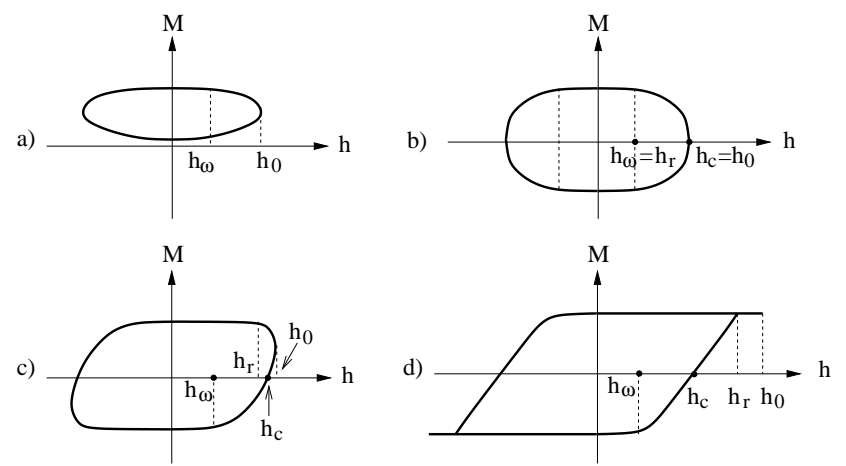

FIG. 2. Schematic pictures of hysteresis loops(HL). (a) Incomplete HL for $h_{0}<h_{t 1}$. (b) Symmetric HL for $h_{0}=h_{t 1}$. (c) The HL for $h_{t} 1<h_{0}<h_{t 2}$. (d) The HL for $h_{0}>h_{t 2}$. The values $h_{p}, h_{c}, h_{r}$ and $h_{0}$ are all marked in all figures

We start with large temperatures $T>T_{p}$ at which the zero-temperature threshold field $h_{p}$ plays no role (field sweep (2) in Fig.1). In this case the motion of the domain wall is determined by equation (13) for $h>h_{\omega}$. As in the case of zero temperature 10 three dynamic phase transitions take place when the amplitude $h_{0}$ increases gradually at a fixed value of frequency. These transitions change the shape (symmetry) of the hysteresis loop. At the first of them proceeding at $h_{0}=h_{\omega}$ the hysteresis loop first appears, at smaller amplitudes $h_{0}<h_{\omega}$ the magnetization remains unchanged. The hysteresis loop appearing at $h_{0}>h_{\omega}$ is characterized by incomplete magnetization reversal and reflection symmetry $h \rightarrow-h, M \rightarrow M$ as shown in Fig. 2a. This symmetry as well as incomplete magnetization reversal persists until the next dynamic phase transition at $h_{0}=h_{t 1}$.

At $h>h_{t 1}$ the magnetization reversal becomes complete and hysteresis loop symmetry changes to inversion $h \rightarrow-h, M \rightarrow-M$ (see Figs. 2b, 2c). The value $h_{t 1}$ is determined by a requirement that the domain wall proceeds from one sample boundary to another for half a period. At the next dynamic phase transition the symmetry of the hysteresis loop remains unchanged, but the part of the cycle becomes reversible. Visually the hysteresis loop acquires characteristic "whiskers" as shown in Fig. 2d. The point of this transition $h_{t 2}$ is determined by a requirement that the domain wall proceeds from one sample boundary to another for quarter period. 
Starting from the transition amplitude $h_{0}=h_{t 1}$ each hysteresis loop goes through three important points. One of them is $h_{\omega}$, at which the motion of domain wall starts. Two others the so-called coercive field $h_{c}$ and reversal field $h_{r}$. At coercive field the magnetization turns into zero, at reversal field the magnetization becomes completely reversed (see Fig. 2b-2d). Note that for $h_{0}=h_{t 1}$ $h_{r}=h_{\omega}$ and $h_{c}=h_{0}$; for $h_{0}=h_{t 2} h_{r}=h_{0}$. All these fields can be found in our case. Equations for $h_{t 1}, h_{t 2}$ are:

$$
\int_{h_{\omega}}^{h_{t n}} \frac{v(h) d h}{\sqrt{h_{t n}^{2}-h^{2}}}=\frac{n \omega L}{2}, n=1,2
$$

For the case $T>T_{p}$ the equations (14) read:

$$
g\left(x_{n}\right)=\frac{n \omega L}{2 \gamma h_{T}}, g(x)=\int_{x_{\omega}}^{x} y e^{-y^{-\mu}}\left(x^{2}-y^{2}\right)^{-1 / 2} d x
$$

where $x_{n}=h_{t n} / h_{T}, n=1,2 ; x_{\omega}=h_{\omega} / h_{T}=1 / \Lambda^{1 / \mu}$. Thus, $x_{1}, x_{2}$ are functions of a dimensionless parameter $u=\omega L / \gamma h_{T}$, where $L$ is the size of the system or an average size of domains. Its asymptotic at small $u$ results in $x_{n} \approx[\ln (2 / n u)]^{-1 / \mu}$. The fields $h_{t 1} ; h_{t 2}$ are close in this case: $\left(h_{t 2}-h_{t 1}\right) / h_{t 1} \approx \ln 2 /(\mu \ln u)$. The requirement $h_{t 1}>h_{\omega}$ is satisfied if $\gamma h_{T} \tau_{0}<L$. The coercive field $h_{c}$ and the reversal field $h_{r}$ are determined by equations: $M\left(h_{c}\right)=0 ;\left|M\left(h_{r}\right)\right|=M_{s}$.

A simple analysis at small $u$ results in $h_{c} \approx h_{t 1} ; h_{r} \approx$ $h_{t 2}$. The area $\mathcal{A}$ of the hysteresis loop at $u<<1$ and $h_{0}>h_{t 1}$ does not depend strongly on the amplitude $h_{0}$ and becomes $\mathcal{A} \approx 4 h_{r} \mathcal{M}_{s}$. Fig.2 shows typical hysteresis loops and illustrates the geometrical meaning of the field $h_{\omega}, h_{c}, h_{r}$. The dependence of magnetization on magnetic field is given by equation: $M(h)=M_{s}\left(\frac{2 Z(h)}{L}-1\right)$.

Finally, in the range of moderately low temperature $T<T_{p}$ the more complete expression (10) and (11) have to be used in integrating equation (14).

It interesting to note that the similar (dynamic) transition from incomplete to complete hysteresis was observed even in a standard simplistic mean-field model for pure magnets with the reaction described by the Brilloin function [12]. This suggests that this kind of dynamic transition we discuss, may be a generic property of nonlinear systems. Another note is in order: hysteresis in large multidomain magnet samples is a very complex phenomenon and cannot be always reduced to motion of a single domain wall (see for example numerical simulations of random Ising model in [11, where hysteretic and memory efects unlikely reducible to motion of a sible DW were revealed).

In conclusion, we have investigated critical creep motion at low, $T \ll T_{p}$, and at high, $T>T_{p}$, temperatures, $T_{p}$ being the depinning temperature, and constructed the dynamic phase diagram. At low temperatures creep at $h \approx h_{p}$ retains features of the critical behavior and exhibits the rounding of the $h-v$ characteristic, according to [17. At finite frequencies $\omega$, a new characteristic field $h_{\omega}<h_{p}$ comes into play, and the transition from the sliding regime to pinning dominated activation motion is shifted to $h_{\omega}$.

Acknowledgements. We are grateful to A. Middleton for a valuable discussion. One of us (V.P.) acknowledges the support of this work by the NSF under the grant DMR-97-05182, DMR-00-72115 and by the DOE under the grant DE-FG03-96ER45598 as well as from Humboldt-foundation. The work at ANL was supported by the by the U.S. DOE, Office of Science under contract No. W-31-109-ENG-38.

[1] for recent reviews see e.g. M. Kardar, Phys. Rep. 301, 85 (1998), D.S. Fisher, Phys. Rep. 301, 113 (1998),

[2] L. B. Ioffe and V.M. Vinokur, J. Phys. C 20, 6149 (1987).

[3] T. Nattermann, Phys. Rev. Lett. 64, 2454 (1990).

[4] M.V. Feigelman, D.B. Geshkenbein, A.I. Larkin, and V.M. Vinokur, Phys. Rev. Lett. 63, 2303 (1989).

[5] G. Blatter, M.V. Feigelman, V.B. Geshkenbein, A.I. Larkin, and V.M. Vinokur, Rev. Mod. Phys. 66, 1125 (1998);

[6] D.S. Fisher, Phys. Rev. Lett. 50, 1486 (1983).

[7] O. Narayan and D. S. Fisher, Phys. Rev. B 46, 11520 (1992).

[8] T. Nattermann, S. Stepanow, L.H. Tang, H. Leschhorn, J. Physique 2,1483 (1992).

[9] O. Narayan and D.S. Fisher, Phys. Rev. B 48, 7030 (1993).

[10] I.F. Lyuksyutov, T. Nattermann, and V.L. Pokrovsky, Phys. Rev. B 59, 2262 (1999).

[11] J. P. Sethna, K. Dahmen, S. Kartha, J.A. Krumhansl, B.W. Roberts and J. D. Shore, Phys. Rev. Lett. 70, 3347 (1993); K. Dahmen and J. P. Sethna, Phys. Rev. Lett. 71, 3222 (1993) .

[12] B. K. Chakrabarti and M. Acharyya, Rev. Mod. Phys. 71, 847 (1999).

[13] T. Halpin-Healy and Y-C. Zhang, Phys. Rep. 254, 215 (1995).

[14] D. S. Fisher, Phys. Rev. Lett. 56, 1964 (1986).

[15] For a RG-derivation of 7 see L. Radzihovsky, APS March Meeting 1998 and P. Chauve, T. Giamarchi and P. Le Doussal, Europhys. Lett. 44, 110 (1998).

[16] J. Villain, J. Phys. Lett. (Paris) 43, L551 (1982); G. Grinstein and S.-K. Ma, Phys. Rev. Lett. 49, 685 (1982).

[17] A. Middleton, Phys. Rev. Lett. 68, 670 (1992).

[18] L.Roters, A. Hucht, S. Lubeck, U. Nowak, and K.D. Usadel, Phys. Rev. E 60, 5202 (1999), B. Koiller and M.O. Robbins, arXiv:cond-mat/0004183 and references therein.

[19] U. Nowak and K. D. Usadel, Europhys. Lett. 44, 634 (1998). 
[20] H. Ji and M.O. Robbins, Phys. Rev. B44, 2538 (1991).

[21] H. Ji and M.O. Robbins, Phys. Rev. B46, 14519 (1992),
B. Drossel and K. Dahmen, Eur. Phys. J. B3, 485 (1998). 